\title{
Mammary Analogue Secretory Carcinoma of Salivary Gland. A Case Report Emphasizing its Diagnostic Histological, Immunohistochemistry and Molecular Findings
}

\author{
${ }^{1}$ Monalisa Hui, ${ }^{2}$ Shantveer G Uppin, ${ }^{3}$ Vamshi Krishna Thamtam, ${ }^{4}$ Abhiram Kalle
}

\begin{abstract}
Mammary analogue secretory carcinoma is a recently described salivary gland tumor that shares morphologic and genetic characteristics with secretory carcinoma of the breast. Due to its complex and heterlogeneous presentation, most of these tumors were previously labeled histologically as acinic cell carcinoma, mucoepidermoid carcinoma or adenocarcinoma not otherwise specified. Here we present a case of MASC of the parotid gland in a 55-year-old man and discuss the histological, $\mathrm{IHC}$ and molecular features that will facilitate its diagnosis and differentiation from its histologic mimics.
\end{abstract}

Keywords: Histology, Mammary analogue secretory carcinoma, Molecular

How to cite this article: Hui M, Uppin SG, Thamtam VK, Kalle A. Mammary Analogue Secretory Carcinoma of Salivary Gland. A Case Report Emphasizing its Diagnostic Histological, Immunohistochemistry and Molecular Findings. Int J Head Neck Surg 2017;8(4):160-163.

Source of support: Nil

Conflict of interest: None

\section{INTRODUCTION}

Mammary analogue secretory carcinoma (MASC) is a recently described salivary gland tumor that shares morphologic and genetic characteristics with secretory carcinoma (SC) of the breast. ${ }^{1}$ Majority of the cases have been diagnosed retrospectively after re-examining the archived salivary gland tumors. Due to its complex and heterogeneous presentation, most of these tumors were previously labeled histologically as acinic cell carcinoma (AciCC), mucoepidermoid carcinoma (MEC) or adenocarcinoma not otherwise specified (NOS). ${ }^{2}$ As there is a considerable cytological and morphological overlap, appropriate immunohistochemical (IHC) profile and

${ }^{1}$ Assistant Professor, ${ }^{2}$ Professor, ${ }^{3,4}$ Junior Resident

${ }^{1-4}$ Department of Pathology, Nizams Institute of medical sciences, Hyderabad, Telangana, India

Corresponding Author: Shantveer G Uppin, Professor, Department of Pathology, Nizams Institute of Medical Sciences, Hyderabad, Telangana, India, e-mail: drsguppin@yahoo.co.in molecular studies aid in correct categorization of this new entity. Here, in this article, we present a case of MASC presenting as a painful mass in the parotid gland in a 55-year-old man. In this article, we emphasized on the histological, IHC and molecular studies that characterize this tumor and help to differentiate from its histologic mimics.

\section{CASE HISTORY}

A 55-year-old male presented with swelling of the left cheek over past 2 years. It was insidious in onset and slow growing. However, since last 2 months he noticed rapid increase in the size with associated pain. On examination, there was diffuse swelling over the angle of jaw which was mobile and soft to firm on palpation. The overlying skin was unremarkable and there was no facial weakness or paralysis. There was no cervical lymphadenopathy. Laboratory investigations were within normal limits. Fine needle aspiration (FNA) done in an outside hospital was reported as pleomorphic adenoma. Following which, he underwent left superficial parotidectomy with preservation of the facial nerve. The excised mass was grey brown nodular measuring $3 \times 3 \mathrm{~cm}$ which on sectioning was predominantly solid with focal cystic areas. The solid areas were grey white lobulated and glistening separated by thin fibrous strands. On histology, it was diagnosed mucoepidermoid carcinoma. On his referral to our hospital for further management, the slide was reviewed and further ancillary studies were performed on the formalin fixed paraffin-embedded blocks submitted. On microscopy, it was well circumscribed with focally invasive borders with cells arranged in lobules separated by thin fibrous septa. Within the lobules, the cells were arranged in cribriform, papillary pattern and microcystic spaces. The cells were polygonal with abundant vacuolated to pale eosinophilic cytoplasm and oval nuclei having finely granular chromatin. The lumen of the microcystic spaces were filled with mucoid material. Cholesterol clefts with surrounding foreign body giant cells were also seen (Fig. 1). There was no significant mitotic activity or necrosis. Immunohistochemistry was done and the panel included S100, GCDFP-15, DOG 1, p63 


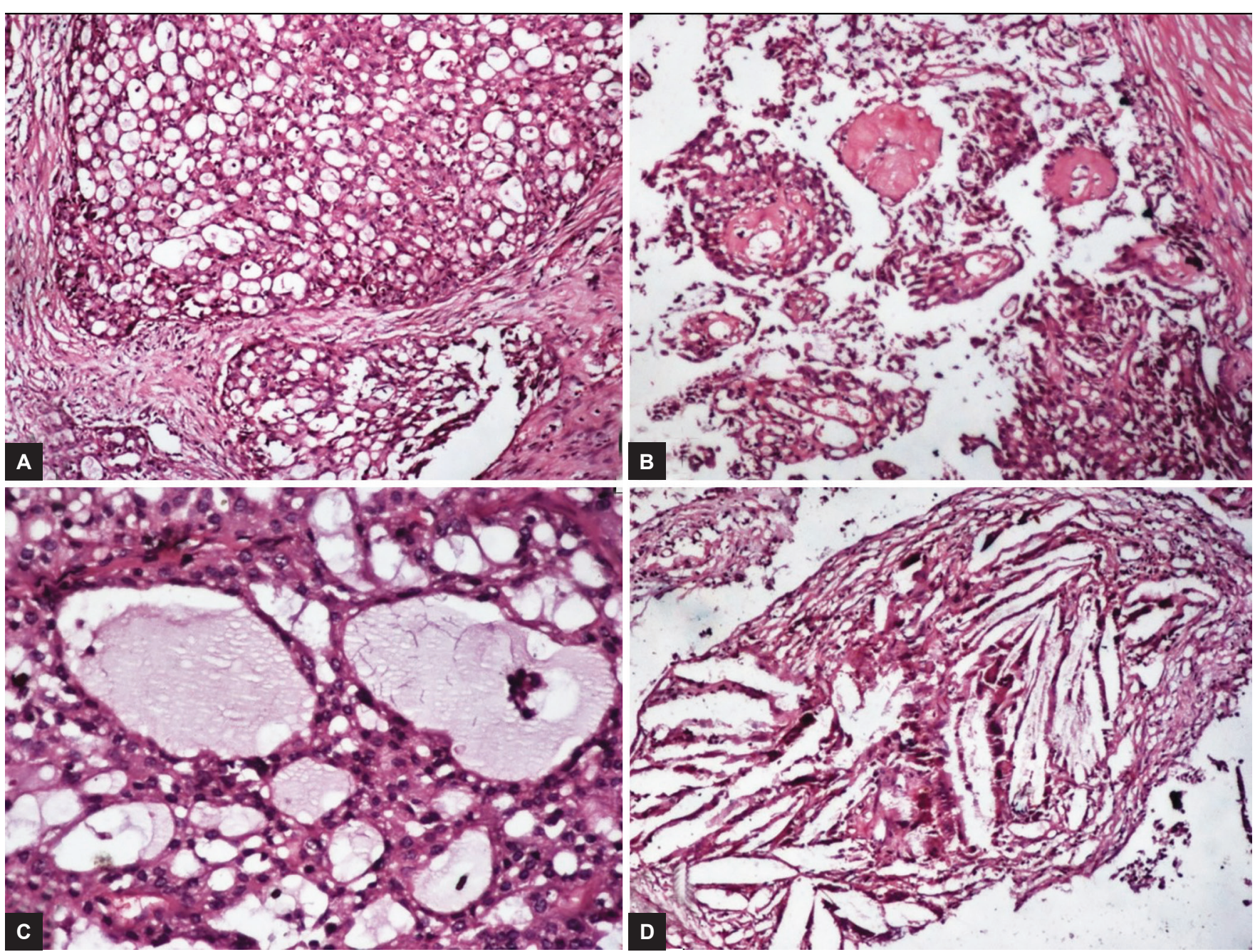

Figs 1A to D: Sections of tumor showing (A) cells arranged in lobules separated by fibrous septae. Cells are polygonal with abundant vacoulated cytoplasm. (B) Areas showing papillary pattern and (C) microcystic spaces. (D) Areas showing cholesterol clefts with surrounding foreign body giant cells. (H\&E; AX40; BX40, CX100 \& DX100)

and EMA. The cells were diffusely positive for S100, EMA and GCDFP-15 (Figs 2A-C). P63 and DOG 1 were negative in the cells and positive in the periphery of the lobules (Figs 2D and E). Based on these histomorphological and IHC features, a possibility of MASC was considered and further molecular analysis with polymerase chain reaction (PCR) based translocation assay was performed to confirm the diagnosis. The presence of characteristic ETV6-NTRK3 fusion confirmed the diagnosis. The patient did not require any further radical surgery or adjuvant therapy and was advised to attend the surgical outpatient department for a close follow-up at regular intervals. He is recurrence free at 8 months postsurgery.

\section{DISCUSSION}

MASC of the salivary gland was first described by Skalova et al. ${ }^{1}$ in 2010. In their series, the authors reclassified 16 cases in which histology and IHC features resembled SC of the breast and subsequently proposed the term MASC to describe these tumors. ${ }^{1}$ MASC encompasses all salivary gland tumors that share identical histologic fea- tures and recurrent balanced chromosomal translocation $\mathrm{t}(12 ; 15)(\mathrm{p} 13 ; \mathrm{q} 25)$ as SC of the breast. ${ }^{3}$ Both the mammary gland and salivary gland shares similar embryological origin and histological organization. ${ }^{4}$ It presents as an asymptomatic mass and most commonly affects parotid gland followed by submandibular gland. ${ }^{5}$ Pain and facial nerve dysfunction are rare. ${ }^{6}$ Bishop et al reported MASC in minor salivary glands of the oral cavity which included lip, soft palate, hard palate, base of tongue and buccal mucosa. In their study, the authors found that almost all extraparotid AciCC corresponded to MASC. ${ }^{7}$ It has a male preponderance with a male:female ratio of 1.5:1. ${ }^{8}$ The mean age of diagnosis is 46 years with a wide range from 21 to 75 years. ${ }^{1}$ Studies have also revealed that the tumor size is smaller in the oral cavity compared to the parotid gland. ${ }^{9}$

The cytological features of MASC has also been described in literature. Studies have shown that there is considerable overlap in the cytological diagnosis of these tumors with AciCC, MEC, salivary duct carcinoma (SDC) and oncocytoma. ${ }^{10}$ Hence, should be considered 


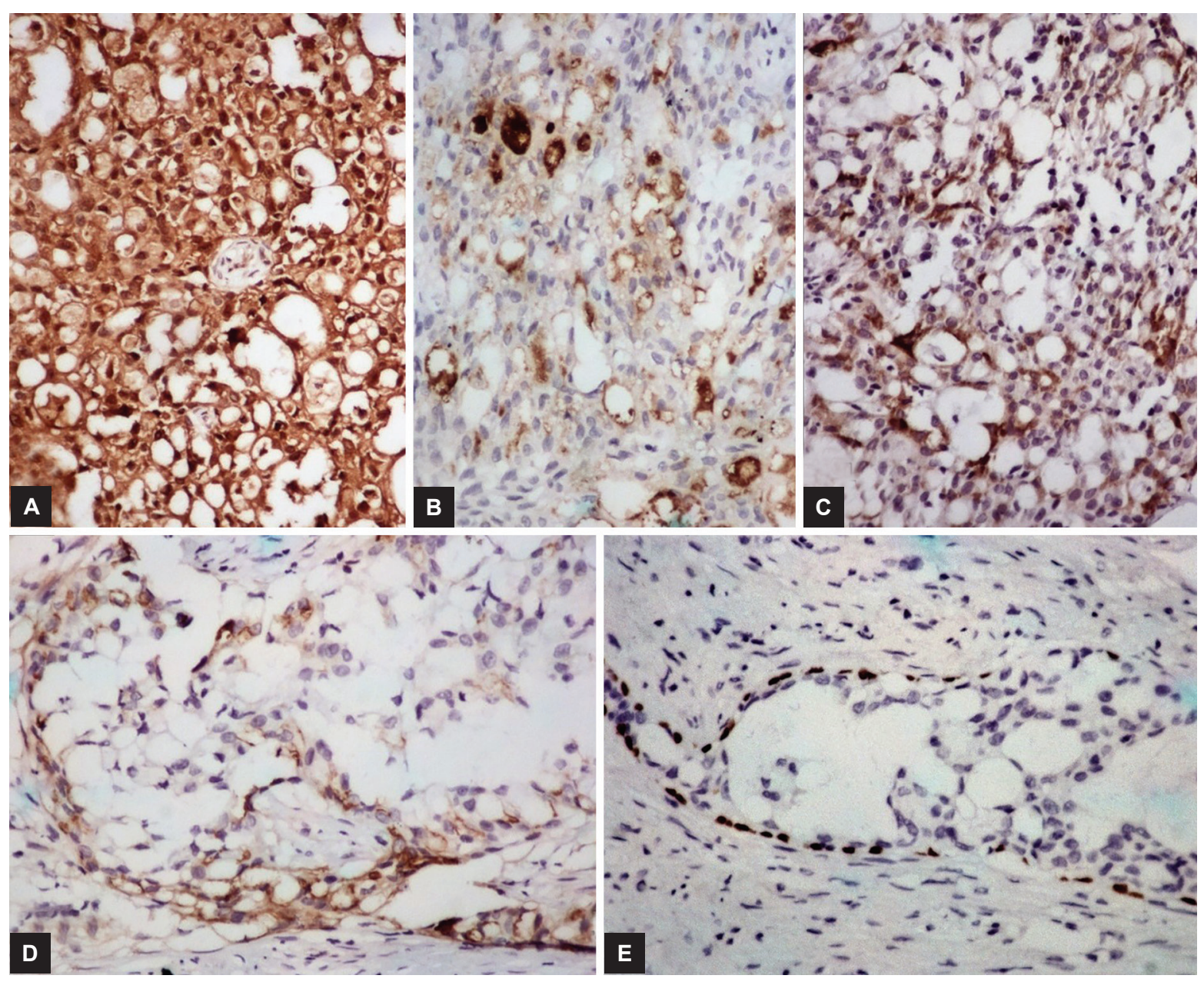

Figs 2A to E: Immunohistochemistry sections showing positivity for (A) S-100; (B) GCDFP-15; (C) EMA; (D) DOG-1; (E) P63 showing staining only at the periphery of lobules where as rest of tumor is negative. (HRP-Polymer; A, B,C,D,E, F X100)

in the differential diagnosis of low grade salivary gland neoplasm. ${ }^{11}$ The present case was misdiagnosed as pleomorphic adenoma on initial FNA. To avoid such misdiagnosis, the secretory material in MASC should be differentiated from the metachromatic fibrillary stroma seen in pleomorphic adenoma and other low grade salivary gland neoplasm. ${ }^{4}$ In addition, cell block preparation from aspirates may be used for IHC and molecular studies.

Histologically both MASC and SC comprises of microcystic, macrocystic and papillary pattern with profileration of cells having pale eosinophilic to vacuolated cytoplasm. The microcystic spaces show PAS-positive secretions within the lumen. ${ }^{4}$ High grade transformation has also been described in literature characterized by nuclear atypia, necrosis and paucity of secretions. ${ }^{12,13}$ IHC similarities includes positivity for S100 protein, epithelial membrane antigen (EMA), and vimentin and are "triple negative" (ER/PR/Her2 negative). ${ }^{14}$ The tumor cells of MASC also show strong and diffuse positivity for mammaglobin, STAT5a, CK7 and are negative for DOG1. Immunostaining for DOG 1 is only restricted to the periphery of the lobules in a non canalicular pattern. ${ }^{3}$
The most important differential diagnosis includes AciCC which is also histologically characterized by microcystic, follicular and papillary patterns. However, unlike the former, MASC does not have acinar cells with cytoplasmic PAS-positive zymogen-like granules. ${ }^{1}$ Hence, majority of MASC were previously diagnosed as 'zymogen-poor' acinic cell carcinoma. ${ }^{8}$ MASC is positive for S100 and negative for DOG1 whereas reverse staining pattern is seen in AciCC. Positive S-100 staining and DOG-1 negativity excluded the possibility of AciCC in our case. The other differential diagnosis include MEC, low-grade cribriform cystadenocarcinoma, SDC and adenocarcinoma NOS. In view of plenty of vacuolated cells with intracytoplasmic secretions, the present case was possibly misdiagnosed outside as MEC. The lack of squamoid areas and negative staining for $\mathrm{p} 63$ (restricted only to the periphery of lobules) exludes MEC in the present case. Cases with high grade transformation can mimic SDC. The expression of androgen receptor or HER-2/neu and negative staining of S100 protein favours SDC over MASC. ${ }^{11}$ Chiosa et al in their review of 337 cases of salivary gland malignancy, re-classified ADC-NOS $(37.6 \%)$, AciCC (12.35\%) and MEC $(0.60 \%)$ as MASC. ${ }^{15}$ 
Although characteristic morphologic and IHC features form the basis of a diagnosis of MASC, the presence of an ETV6-NTRK3 gene fusion is confirmatory. This fusion gene product encodes a chimeric tyrosine kinase that results in cell proliferation and increased cell survival leading to oncogenesis. ${ }^{11}$ Apart from MASC and SC, this fusion gene product is also seen in infantile fibrosarcoma, myelogenous leukemia, and congenital mesoblastic nephroma. ${ }^{16}$ However among the salivary gland tumors, this translocation is specific for MASC and its presence helps to reclassify cases of adenocarcinoma (NOS)/cystadenocarcinoma which are otherwise a diagnosis of exclusion. ${ }^{11}$ Molecular analysis can be done either by FISH using a break-apart probe for the ETV6 gene or by reverse transcription-polymerase chain reaction (RT-PCR) using ETV6 and NTRK3 gene-specific primer. ${ }^{3}$ Majewska et al in their study found that FISH for ETV6 gene rearrangement was positive in six out of seven cases, and RT-PCR was positive in three cases. ${ }^{11}$ Similarly Skalova et al in their study also found that all the cases were positive for ETV6 gene rearrangement on FISH and 13 out of 14 cases were positive for fusion transcript by RT-PCR. ${ }^{1}$ The negative RT-PCR results have been attributed to a different fusion partner for the ETV6 gene or non-recognition of break in the NTRK3 gene by the primers. ${ }^{4}$ The presence of fusion gene product by RT-PCR method was also documented in our case.

MASC is considered to be a low-grade carcinoma with a favourable prognosis. ${ }^{1}$ It has potential for regional lymph node and distant metastasis. The disease free period is shorter and the risk of lymph node involvement is higher in MASC compared to acinic cell carcinoma. In case of incomplete excision, the risk of recurrence is $15 \%{ }^{8}$ Adjuvant radiotherapy is required in recurrent cases and high grade transformation. ${ }^{11}$ It has a more aggressive behavior than its mammary counterpart. ${ }^{4}$

Diagnosis requires a high index of suspicion and awareness of this newly described entity. In cases of morphological ambiguity, the use of appropriate panel of immunomarkers increases the diagnostic accuracy. Molecular studies serve as the final tool to differentiate MASC from its mimickers. Diagnosis and differentiation from other low grade salivary gland tumors is essential as the presence of ETV6-NTRK3 translocation provides a potential therapeutic target in future.

\section{REFERENCES}

1. Skálová A, Vanecek T., Sima R. Mammary analogue secretory carcinoma of salivary glands, containing the ETV6-NTRK3 fusion gene: a hitherto undescribed salivary gland tumor entity. Am. J. Surg. Pathol. 2010;34:599-608.
2. Wang S, Li Y, Li G, Zhu L. Mammary analogue secretory carcinoma presenting as a salivary gland neoplasm: a first clinical case report of an exceptionally rare tumor in China Int J Clin Exp Med 2016;9:22632-22637

3. Stevens TM, Parekh V. Mammary Analogue Secretory Carcinoma. Arch Pathol Lab Med. 2016;140:997-1001.

4. Petersson F, Lian D, Chau YP, Yan B. Mammary analogue secretory carcinoma: the first submandibular case reported including findings on fine needle aspiration cytology. Head Neck Pathol.2012;6:135-139.

5. Levine P, Fried K, Krevitt L. Aspiration biopsy of mammary analogue secretory carcinoma of accessory parotid gland: another diagnostic dilemma in matrix-containing tumors of the salivary glands. Diagn. Citopathol. 2012;42:49-53.

6. Sethi R, Kozin E, Remenschneider A, Meier J, VanderLaan $\mathrm{P}$, Faquin $\mathrm{W}$ et al.Mammary analogue secretory carcinoma: update on a new diagnosis of salivary gland malignancy. Laryngoscope 2014; 124: 188-195.

7. Bishop J, Yonescu R, Bastista D. Most nonparotid actinic cell carcinomas represent mammary analog secretory carcinomas. Am. J. Surg. Pathol. 2013;37:1053-1057.

8. Jackson BS, Pratt TL, Rooyen A Mammary analogue secretory carcinoma: A rare salivary gland tumour. S Afr Med J 2017;107:304-306

9. Bishop JA. Unmasking MASC: bringing to light the unique morphologic, immunohistochemical and genetic features of the newly recognized mammary analogue secretory carcinoma of salivary glands. Head Neck Pathol. 2013;7:35-39

10. Griffith CC, Stelow EB, Saqi A, et al. The cytological features of mammary analogue secretory carcinoma: a series of 6 molecularly confirmed cases. Cancer Cytopathol. 2013;121:234-241

11. Majewska H, Skálová A, Stodulski D, Klimková A, Steiner P, Stankiewicz C, Biernat W. Mammary analogue secretory carcinoma of salivary glands: a new entity associated with ETV6 gene rearrangement. Virchows Arch.2015;466:245-254.

12. Skálová A, Vanecek T, Majewska H. Mammary analogue secretory carcinoma of salivary glands with high-grade transformation: report of 3 cases with the ETV6-NTRK3 gene fusion and analysis of TP53, b-catenin, EGFR, and CCND1 genes. Am J Surg Pathol. 2014;38:23-33.

13. Jung MJ, Song JS, Kim SY, Nam SY, Roh JL, Choi SH, Kim SB and Cho KJ. Finding and characterizing mammary analogue secretory carcinoma of the salivary gland. Korean J Pathol 2013; 47: 36-43

14. Connor A, Perez-Ordoñez B, Shago M. Mammary analog secretory carcinoma of salivary gland origin with the ETV6 gene rearrangement by FISH: expanded morphologic and immunohistochemical spectrum of a recently described entity. Am. J. Surg. Pathol. 2012;36:27-34.

15. Chiosea SI, Griffith C, Assaad A, Seethala RR. Clinicopathological characterization of mammary analogue secretory carcinoma of salivary glands. Histopathology. 2012; 61:387-394

16. Balanzá R, Arrangoiz R, Cordera F, Muñoz M, Luque-deLeón E, Moreno E, Toledo C, González E. Mammary analog secretory carcinoma of the parotid gland: A case report and literature review. Int J Surg Case Rep. 2015;16:187-191. 